Erratum

\title{
Erratum to "Development and Validation of a LC/MS/MS Method for the Determination of Duloxetine in Human Plasma and Its Application to Pharmacokinetic Study"
}

\author{
D. Chandrapal Reddy, ${ }^{1,2}$ A. T. Bapuji, ${ }^{1}$ V. Suryanarayana Rao, ${ }^{3}$ V. Himabindu, ${ }^{2}$ \\ D. Rama Raju, ${ }^{1}$ Syed Syedba, ${ }^{1}$ and H. L. V. Ravikiran ${ }^{1}$ \\ ${ }^{1}$ Department of Clinical Pharmacology, APL Research Centre, Hyderabad 500090, India \\ ${ }^{2}$ Institute of Science and Technology, Jawaharlal Nehru Technological University, Kukatpally, Hyderabad 500085, India \\ ${ }^{3}$ Department of Chemistry, Sri Krishnadevaraya University, Anantapur 515 055, India
}

Correspondence should be addressed to D. Chandrapal Reddy; dcpreddy007@yahoo.co.in

Received 3 July 2012; Accepted 25 September 2012

Copyright (c) 2013 D. Chandrapal Reddy et al. This is an open access article distributed under the Creative Commons Attribution License, which permits unrestricted use, distribution, and reproduction in any medium, provided the original work is properly cited.

The typo error happened in Table 2 has now been modified as mentioned below. 
TABLE 2: Absolute matrix effect, relative recovery, and process efficiency for duloxetine.

\begin{tabular}{|c|c|c|c|c|c|c|}
\hline $\begin{array}{l}\text { Analyte } \\
\text { ISTD }\end{array}$ & $A^{\mathrm{a}}(\% \mathrm{CV})$ & $B^{\mathrm{b}}(\% \mathrm{CV})$ & $C^{c}(\% \mathrm{CV})$ & Matrix effect $(\% \mathrm{ME})^{\mathrm{d}}$ & Recovery $(\% \mathrm{RE})^{\mathrm{e}}$ & Process efficiency $(\% \mathrm{PE})^{\mathrm{f}}$ \\
\hline \multicolumn{7}{|l|}{ STD 1} \\
\hline Duloxetine & $170(4.86)$ & $160(3.75)$ & $136(4.32)$ & 94.23 & 85.40 & 80.47 \\
\hline Fluoxetine & $106783(2.34)$ & 96545 (2.99) & $81421(1.32)$ & 90.41 & 84.34 & 76.25 \\
\hline \multicolumn{7}{|l|}{ STD 3} \\
\hline Duloxetine & $708(3.23)$ & $739(4.13)$ & $575(1.22)$ & 104.38 & 77.77 & 81.18 \\
\hline Fluoxetine & $104735(1.31)$ & $108227(1.56)$ & $81902(1.80)$ & 103.33 & 75.68 & 78.20 \\
\hline \multicolumn{7}{|l|}{ STD 5} \\
\hline Duloxetine & $57893(1.74)$ & $57170(1.69)$ & $45749(0.96)$ & 98.75 & 80.02 & 79.02 \\
\hline Fluoxetine & $100839(1.94)$ & $98731(1.65)$ & $82613(0.68)$ & 97.91 & 83.67 & 81.93 \\
\hline \multicolumn{7}{|l|}{ STD 6} \\
\hline Duloxetine & $174918(1.09)$ & $172409(1.24)$ & $138067(1.01)$ & 98.57 & 80.08 & 78.93 \\
\hline Fluoxetine & $95399(1.53)$ & $94770(1.56)$ & 76090 (1.07) & 99.34 & 80.29 & 79.76 \\
\hline \multicolumn{7}{|l|}{ STD 8} \\
\hline Duloxetine & $349836(1.09)$ & $344817(1.24)$ & $269986(6.06)$ & 98.57 & 78.30 & 77.18 \\
\hline Fluoxetine & $85599(1.61)$ & $87554(2.23)$ & $71346(0.67)$ & 102.28 & 81.49 & 83.35 \\
\hline
\end{tabular}

aean area response of six replicate samples prepared in mobile phase (neat samples).

${ }^{b}$ Mean area response of six replicate samples prepared by spiking in postextracted blank.

${ }^{\mathrm{c}}$ Mean area response of six replicate samples prepared by spiking in plasma before extraction.

$\mathrm{d}_{\%}$ Matrix effect: postextracted mean response/aqueous neat mean response $\times 100$.

$\mathrm{e}_{\%}$ Recovery: extracted mean response/postextracted mean response $\times 100$.

$\mathrm{f}_{\%}$ Process efficiency: extracted mean response/aqueous mean response $\times 100$. 

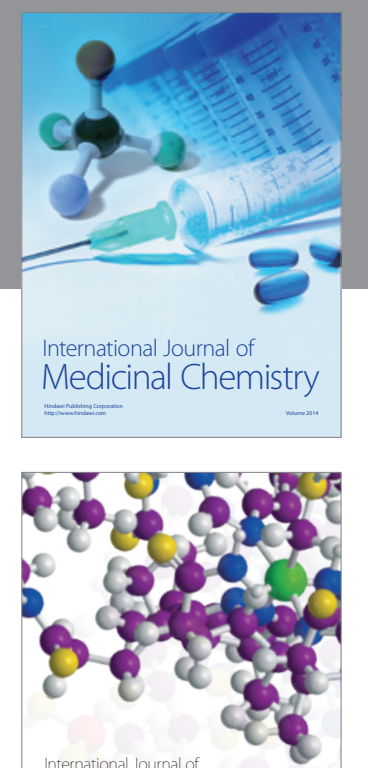

\section{Carbohydrate} Chemistry

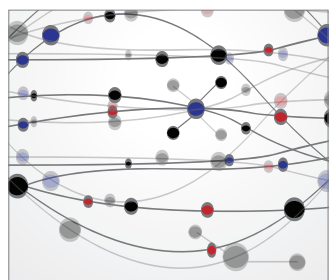

The Scientific World Journal
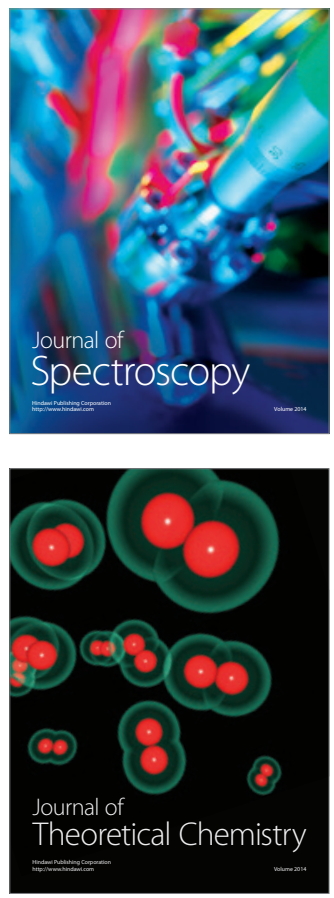
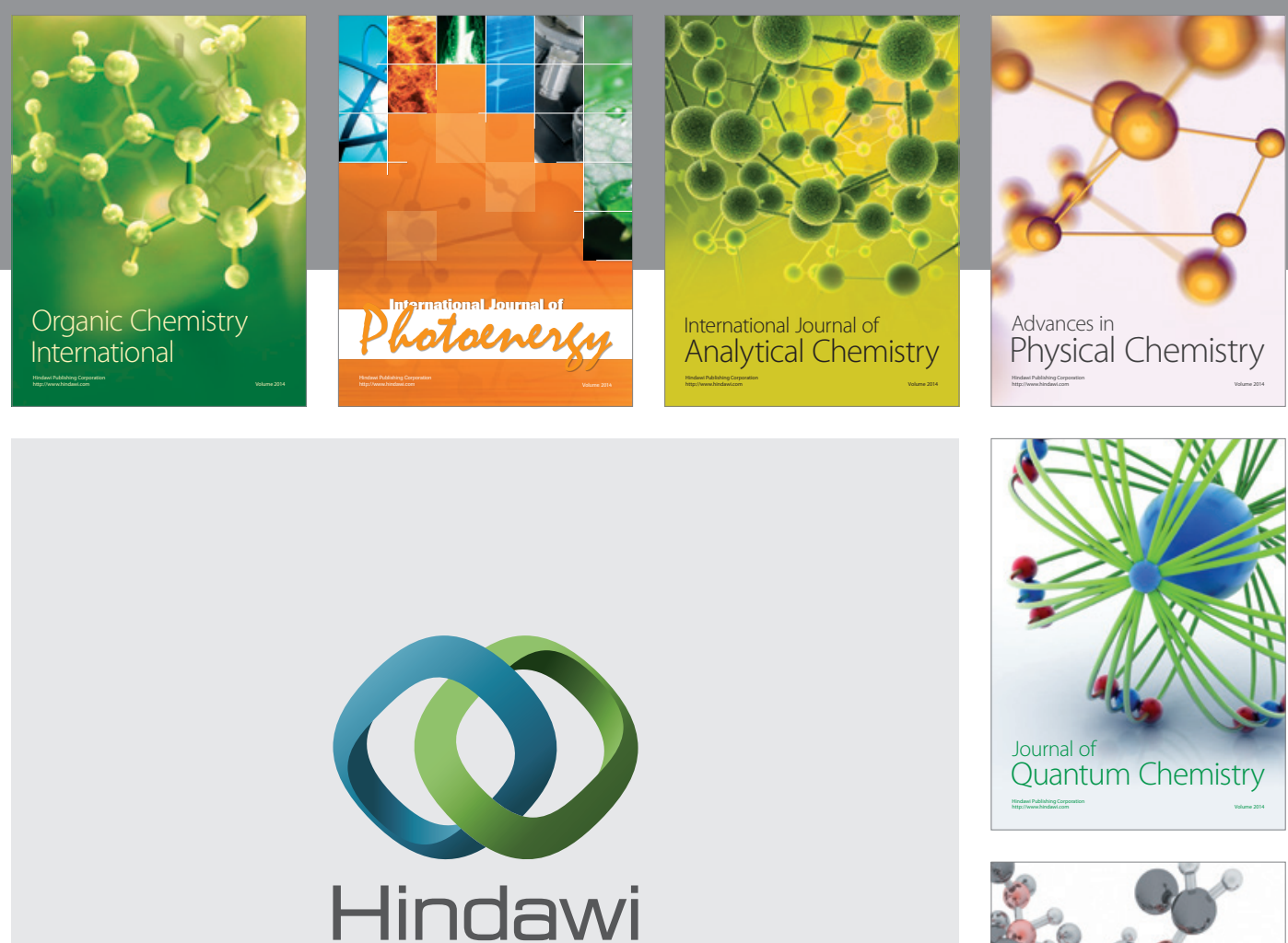

Submit your manuscripts at

http://www.hindawi.com

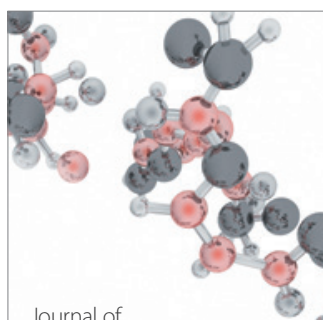

Analytical Methods

in Chemistry

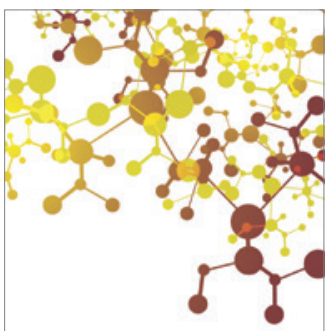

Journal of

Applied Chemistry

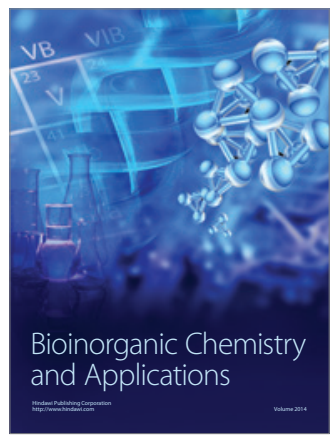

Inorganic Chemistry
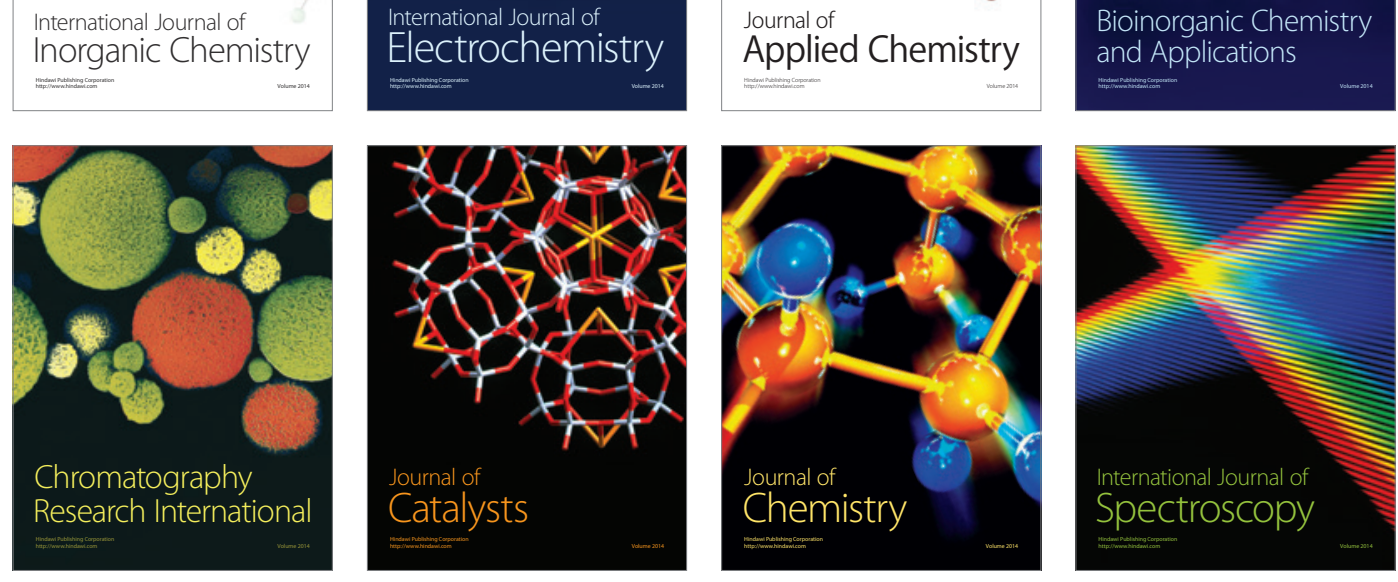